\title{
G250 Antigen-Targeting Drug-Loaded Nanobubbles Combined with Ultrasound Targeted Nanobubble Destruction: A Potential Novel Treatment for Renal Cell Carcinoma
}

This article was published in the following Dove Press journal:

International Journal of Nanomedicine

\section{Zhiping $\mathrm{Yu}^{\prime}$ \\ Yixuan Wang $\mathbb{D}^{2}$ \\ Dan $X u^{3}$ \\ Lianhua Zhu (iD) \\ Ming $\mathrm{Hu}$ (D) $^{\prime}$ \\ Qiuli Liu' \\ Weihua Lan' \\ Jun Jiang' \\ Luofu Wang (D)'}

'Department of Urology, Daping Hospital, Army Medical University, Chongqing, People's Republic of China; ${ }^{2}$ The First Clinical College, Chongqing Medical University, Chongqing, People's Republic of China; ${ }^{3}$ Department of Ultrasound, Southwest Hospital, Army Medical University, Chongqing, People's Republic of China
Correspondence: Luofu Wang

Department of Urology, Daping Hospital, No. 10 Changjiangzhilu Road, Yuzhong

District, Chongqing 400042, People's

Republic of China

Tel +86- 13320350708

Fax +86-23-68757947

Email wangluofu@aliyun.com
Purpose: We intended to design G250 antigen-targeting temsirolimus-loaded nanobubbles (G250-TNBs) based on the targeted drug delivery system and to combine G250-TNBs with ultrasound targeted nanobubble destruction (UTND) to achieve a synergistic treatment for renal cell carcinoma (RCC).

Methods: The filming-rehydration method was combined with mechanical shock and electrostatic interactions to prepare temsirolimus-loaded nanobubbles (TNBs). G250-TNBs were prepared by attaching anti-G250 nanobodies to the surface of TNBs using the biotinstreptavidin-bridge method. The ability of G250-TNBs to target the G250 antigen of RCC cells and the synergistic efficacy of G250-TNBs and UTND in the treatment of RCC were assessed.

Results: The average diameter of the prepared G250-TNBs was $368.7 \pm 43.4 \mathrm{~nm}$, the encapsulation efficiency was $68.59 \% \pm 5.43 \%$, and the loading efficiency was $5.23 \% \pm$ $0.91 \%$. In vitro experiments showed that the affinity of G250-TNBs to the human RCC 786$\mathrm{O}$ cells was significantly higher than that of TNBs $(\mathrm{P}<0.05)$, and the inhibitory effect on $786-\mathrm{O}$ cell proliferation and the induction of 786-O cell apoptosis was significantly enhanced in the group treated with G250-TNBs and UTND (G250-TNBs+ UTND group) compared with the other groups $(\mathrm{P}<0.05)$. In a nude mouse xenograft model, compared with TNBs, G250-TNBs could target the transplanted tumors and thus significantly enhance the ultrasound imaging of the tumors. Compared with all other groups, the G250-TNBs+UTND group exhibited a significantly lower tumor volume, a higher tumor growth inhibition rate, and a higher apoptosis index $(\mathrm{P}<0.05)$.

Conclusion: The combined G250-TNBs and UTND treatment can deliver anti-tumor drugs to local areas of RCC, increase the local effective drug concentration, and enhance antitumor efficacy, thus providing a potential novel method for targeted therapy of RCC.

Keywords: nanobubble, G250 antigen, nanobody, targeted drug delivery system, renal cell carcinoma, temsirolimus

\section{Introduction}

Renal cell carcinoma (RCC) is the most common renal malignancy. It is insensitive to both chemotherapy and radiotherapy. Partial or radical resection can improve the prognosis of early-stage RCC patients. However, RCC is difficult to detect early, and the incidence of postoperative recurrence and distant metastasis is high, reducing the survival prognosis of RCC patients. ${ }^{1}$ With the development of molecular 
medicine, molecular targeted drugs play a stronger antitumor role while reducing the toxic and side effects on normal cells. ${ }^{2}$ Temsirolimus (TEM), a molecular targeted drug, is an inhibitor of mammalian target of rapamycin (mTOR). The guidelines of the National Comprehensive Cancer Network (NCCN) and the European Association of Urology (EAU) both recommend TEM as a first-line treatment for patients with metastatic RCC (mRCC) who have high scores for risk factors. ${ }^{3}$ However, due to dose-limiting side effects, the effective concentration of TEM in target tissues is not high enough, and systemic administration has considerable toxic and side effects, thereby affecting the prognosis of RCC. ${ }^{4}$ As a result, safer and more effective targeted therapies continue to be demanded in clinical treatment to increase the effective drug concentration in specific target tissues, thereby improving the treatment efficacy and reducing side effects. The targeted drug delivery system (TDDS) is a possible approach to solve this problem.

Existing studies have already reported TDDSs for the targeted therapy of renal cancer. First, G250 antigen, which is widely expressed in renal cancer cells, can be used as an effective target in TDDSs. Studies have shown that $\mathrm{G} 250$ antigen is a membrane protein that is expressed in approximately $90 \%$ of RCC and is not expressed in normal kidney tissues. G250 antigen is known as renal cancer-associated antigen and can be used as a target for renal cancer therapy. ${ }^{5}$ In our previous experimental study, camel-derived anti-G250 nanobodies that could specifically bind to G250 antigen were prepared by phage display technology. ${ }^{6}$ Compared with monoclonal antibodies, antiG250 nanobodies have a smaller molecular weight (15-20 $\mathrm{kDa}$ ), reduced immunogenicity and higher specificity. ${ }^{7}$ They provide an ideal ligand for targeted therapy of RCC and can bind to targets by linking to drug-loaded particles in a TDDS.

Second, the application of lipid nanobubbles (NBs) has gradually matured. NBs can enhance the contrast of tissue ultrasonography and can be used for the diagnosis of tumors. NBs also provide safe and effective carriers for drugs and genes ${ }^{8,9}$ and can be used as important components in TDDSs. Zhu et al and Yang et al used the biotinstreptavidin-bridge method to link specific ligands of the targeted tumor cells to the surfaces of NBs to achieve active targeting of TDDS. ${ }^{10,11}$ In addition, tumor blood vessels were observed to allow NBs with diameters smaller than $700 \mathrm{~nm}$ to enter the tumor tissue space through the enhanced permeability and retention (EPR) effect. ${ }^{12}$ So
TDDS based on targeted nanobubble can deliver drugs to a specific target site under the joint action of the active targeting effect and the EPR effect. Ultrasound can locally release drug-loaded NBs under ultrasound-targeted nanobubble destruction (UTND), which not only increases the local drug concentrations but also reduces toxic and side effects caused by systemic administration and improves antitumor efficacy, maximizing drug efficacy and minimizing toxic and side effects. ${ }^{13,14}$

Based on the above research, this study designed G250 antigen-targeting (anti-G250 nanobody-carrying) temsirolimus-loaded nanobubbles (G250-TNBs) to verify the targeting effect of this TDDS on renal cancer cells, to investigate the inhibitory effect of G250-TNBs and UTND on the growth of renal cancer cells in vitro, and to examine the therapeutic effect of G250-TNBs and UTND on RCC xenografts in nude mice in vivo.

\section{Materials and Methods \\ Cell Culture}

The RCC cell line 786-O (expressing G250 antigen) was purchased from Shanghai Institute of Biochemistry and Cell Biology, Chinese Academy of Sciences, Shanghai, China. The cells were cultured in RPMI1640 (Biological Industries Ltd, Kibbutz Beit-Haemek, Israel) containing 10\% FBS (Biological Industries Ltd, Kibbutz BeitHaemek, Israel) (v/v), penicillin $(100 \mathrm{U} / \mathrm{mL})$, and streptomycin $(100 \mu \mathrm{g} / \mathrm{mL})$ and incubated at $37^{\circ} \mathrm{C}, 5 \% \mathrm{CO}_{2}$. When the cells were in the logarithmic growth phase, the cells were digested with $0.25 \%$ trypsin for subsequent experiments. The number of cells was counted using a hemocytometer.

\section{Establishment of the RCC Xenograft Model in Nude Mice}

Balb/c nude mice (4 weeks, 15-20 g each) were purchased from Beijing HFK Bio-Technology Co., Ltd. (Beijing, China). All nude mice were housed in a specific-pathogenfree (SPF) environment and allowed access to food and water ad libitum. The $786-\mathrm{O}$ cells $\left(1 \times 10^{7}\right.$ cells $\left./ 200 \mu \mathrm{L}\right)$ were resuspended in matrigel and inoculated into the dorsal subcutaneous fat pads of nude mice. After inoculation, the mice were maintained for 20 days under SPF conditions, with observation, weighing, and tumor size measurements every 3 days. Tumor length (L) and width (W) were measured using a Vernier caliper, and the tumor volume was calculated using the formula $\mathrm{V}=\mathrm{L} \times \mathrm{W}^{2} / 2$. Twenty days after tumor 
inoculation, the average tumor volume reached $50-100 \mathrm{~mm}^{3}$. Subsequent experiments were then conducted. The animal experiments in this study were performed in accordance with NC3Rs ARRIVE guidelines and were approved by the Laboratory Animal Welfare and Ethics Committee of the Army Medical University.

\section{Preparation of Targeted Drug-Loaded NBs}

First, 1,2-dipalmitoyl-sn-glycero-3-phosphocholine (DPPC), 1,2-dipalmitoyl-SN-glycero-3-phosphoethanolamine (DPPE), 1,2-dipalmitoyl-SN-glycero-3-phosphoglycerol (DPPG), and 1,2-dipalmitoyl-sn-glycero-3-phosphate (DPPA) (Corden Pharma, Liestal, Switzerland) and biotin-modified 1,2-distearoyl-sn-glycero-3-phosphoethanomamine-PEG2000 (DSPEPEG2000-Biotin)(NANOCS, Boston, MA, USA) were mixed at a 3:3:3:1:1 mass ratio. A total of $11 \mathrm{mg}$ of the lipid mixture and $2 \mathrm{mg}$ of temsirolimus (TEM) (purity: 99.14\%) (Selleck Chemicals, Houston, Texas, USA) were dissolved in chloroform and anhydrous ethanol (1:1). The solution was transferred into a round-bottom flask, and the solvent was removed by rotary evaporation at $55^{\circ} \mathrm{C}$ under a vacuum. The resulting lipid membrane was hydrated in $1 \mathrm{~mL}$ mixed solution of glycerin and phosphate-buffered saline (PBS) (1:9 volume ratio), transferred to a penicillin bottle, and sealed. After replacing the air in the bottle with octafluoropropane $\left(\mathrm{C}_{3} \mathrm{~F}_{8}\right.$, Tianjin Nuclear Industry Institute of Physics and Chemistry, Tianjin, China), the bottle was placed in the ST-B series mixer (AT \& M Biomaterials Co., Beijing, China) for horizontal reciprocating mechanical oscillation for $90 \mathrm{~s}$. The cells were centrifuged at $300 \mathrm{~g}$ and $300 \mathrm{r} / \mathrm{min}$ each for $3 \mathrm{~min}$ to remove the free lipid components and unencapsulated drugs to obtain the TNBs. Dillabeled TNBs were prepared by adding $5 \mu \mathrm{L}$ 1,1'-dioctadecyl-3,3,3',3'-tetramethylindocarbocyanine perchlorate (Dil) (a fluorescent dye, Beyotime Biotechnology, Shanghai, China) to the lipid solution in the dark. Then, $3 \mu \mathrm{g}$ of streptavidin (SA) (Solarbio, Beijing, China) was added for every $1 \times 10^{7} \mathrm{NBs}$, incubated at $4^{\circ} \mathrm{C}$ for $1 \mathrm{hr}$ and washed 3 times to remove free $\mathrm{SA} ; 0.64 \mu \mathrm{g}$ of biotinylated anti-G250 nanobodies was added for every $1 \times 10^{7} \mathrm{NBs}$ at $4^{\circ} \mathrm{C}$ overnight and washed 3 times to remove the free antibodies. Thus, G250-TNBs were obtained.

\section{Characterization of G250-TNBs}

The distribution of G250-TNBs was observed using an IX71 optical microscope (Olympus Corporation, Kyoto,
Japan), and the concentration was measured using a hematocytometer. Morphological characteristics were observed using a JEM-1400 transmission electron microscope (JEOL, Tokyo, Japan). The mean particle size, polydispersity index (PDI), and zeta potential were measured using a Malvern Zetasizer Nano ZS90 (Malvern Instruments Inc., Worcestershire, UK). The G250-TNBs were observed and measured daily over a period of 1 week to evaluate changes in concentration and particle diameter with time (during this time, they were stored at $4^{\circ} \mathrm{C}$ ). The encapsulation efficiency (EE) and loading efficiency (LE) of G250-TNBs were measured by an Agilent 1260 highperformance liquid chromatography (HPLC) (Agilent Technologies, Santa Clara, CA, USA). The chromatographic conditions were as follows: chromatographic column: Zorbax Eclipse XDB-C18 (150 mm × $4.6 \mathrm{~mm}, 3$ $\mu \mathrm{m}$ ); column temperature: $30^{\circ} \mathrm{C}$; flow rate: $0.8 \mathrm{~mL} / \mathrm{min}$; injection volume: $10 \mu \mathrm{L}$; mobile phase: acetonitrile: $0.5 \%$ glacial acetic acid (45:55); detection wavelength: $277 \mathrm{~nm}$. $\mathrm{EE}=$ amount of TEM encapsulated in NBs/total amount of added TEM $\times 100 \%$; LE $=$ amount of TEM encapsulated in NBs/total amount of lipids for NB preparation $\times$ $100 \%$. $^{15}$

\section{Detection of the Coupling of Anti-G250 Nanobodies to TNBs by Immunofluorescence}

Dil-labeled G250-TNB samples $(200 \mu \mathrm{L})$ were resuspended in $200 \mu \mathrm{L}$ PBS-diluted (1:50) anti-HA antibody (secondary antibody, Proteintech, Wuhan, Hubei, China), mixed well, and reacted on ice for 45 mins. Then, $200 \mu \mathrm{L}$ of PBS-diluted (1:200) goat anti-mouse Alexa-488 (Alexa Fluor $^{\circledR}$ 488, Abcam, Cambridge, UK) was added in the dark and reacted on ice for 45 mins. Ten microliters of the samples were diluted and observed under a Zeiss 780 confocal laser scanning microscope (CLSM) (Carl Zeiss AG, Oberkochen, Germany).

\section{In vitro Drug Release}

The drug release efficiency of G250-TNBs under ultrasound irradiation was evaluated using the dialysis bag method. G250-TNBs were loaded into the dialysis bag, and the two ends of the dialysis bag were sealed. Subsequently, the dialysis bag was completely immersed in a sustained-release medium containing $50 \mathrm{~mL} \mathrm{PBS}$, and oscillation dialysis was performed under $(37.0 \pm 0.5){ }^{\circ} \mathrm{C}$ at $60 \mathrm{rpm}$. The bags were divided into two groups, one of which was randomly selected 
to receive ultrasonic irradiation at $2 \mathrm{hrs}$ (parameter: frequency $1 \mathrm{MHz}$, power $2.5 \mathrm{~W} / \mathrm{cm}^{2}$, and duration $5 \mathrm{mins}$ ) using a WED-100 full digital ultrasonic therapy machine (Shenzhen WELLD Medical Electronics, Shenzhen, China). At 2 hrs, 4 hrs, 6 hrs, 12 hrs, 24 hrs, 48 hrs, and 72 hrs, $1 \mathrm{~mL}$ dialysate from the external solution of the dialysis bag was collected and replaced with $1 \mathrm{~mL}$ of fresh PBS. The amount of TEM released into the dialysate was quantified using the HPLC system at $227 \mathrm{~nm}$, the standard curve was generated, and the percentage of released TEM was calculated.

\section{In vitro Targeted Binding Ability of G250- TNBs}

After an appropriate amount of 786-O cells was cultured in 24-well plates with cell climbing slices and incubated at $37^{\circ}$ C, $5 \% \mathrm{CO}_{2}$ for $12 \mathrm{hrs}$, the cells were washed 3 times with PBS. Cells were fixed in 4\% paraformaldehyde and blocked with PBS containing 5\% bovine serum albumin (BSA-PBS). Subsequently, 786-O cells were divided into three groups: the first group was incubated with Dil-labeled G250-TNBs, the second group was incubated with Dil-labeled TNBs, and in the third group, 786-O cells were cocultured with excess anti-G250 nanobodies for $2 \mathrm{hrs}$ before the addition of Dillabeled G250-TNBs. In all groups, either $50 \mu \mathrm{L}$ of Dillabeled G250-TNBs $\left(1 \times 10^{7} / \mathrm{mL}\right)$ or $50 \mu \mathrm{L}$ of Dil-labeled TNBs $\left(1 \times 10^{7} / \mathrm{mL}\right)$ was added. After incubation at $4^{\circ} \mathrm{C}$ for 2 hrs, the cells were washed 3 times with PBS and stained with 3,3'-dioctadecyloxacarbocyanine perchlorate (DiO) and 4',6-diamidino-2-phenylindole (DAPI) (Beyotime Biotechnology, Shanghai, China) for observation of the cell membranes and nuclei. The stained coverslips were imaged using CLSM. Dil, DiO, and DAPI were observed in red, green, and blue colors, respectively.

\section{Detection of the Cytotoxicity of G250- TNBs in Combination with Ultrasonic Irradiation}

The CCK-8 assay was used to assess the cytotoxicity of G250-TNBs in combination with ultrasound. The concentration of 786-O cells in the logarithmic growth phase was adjusted to $5 \times 10^{3} / \mathrm{mL}$. Cell suspensions $(100 \mu \mathrm{L})$ were inoculated into each well of a 96-well plate and cultured overnight in an incubator. Cells were divided into 6 groups: control group, group directly treated with TEM alone (TEM group), group treated with TNBs alone (TNB group), group treated with G250-TNBs alone (G250-TNBs group), group treated with TNBs and ultrasonic irradiation (TNBs+UTND group), and group treated with G250TNBs and ultrasonic irradiation (G250-TNBs+UTND group). The control group did not receive any treatment. Except for the control group, the same dose of TEM was used for the other groups, and the final concentration was $2 \mathrm{mM}$. Parameters for ultrasonic irradiation were as follows: frequency of $1 \mathrm{MHz}$, irradiation intensity of 1.0 $\mathrm{W} / \mathrm{cm}^{2}$, and irradiation time of $30 \mathrm{~s} /$ well. After $24 \mathrm{hrs}$ of treatment, the fresh medium was replaced, and each well was supplemented with $10 \mu \mathrm{L}$ CCK-8 reagent (Beyotime Biotechnology, Shanghai, China) and cultured in an incubator for $1 \mathrm{hr}$. The optical density (OD) was read at $450 \mathrm{~nm}$ using a microplate reader (Thermo Fisher Scientific, Waltham, MA, USA), and cell viability (\%) was calculated using the following equation: cell viability $=(A s-A b) /(A c-A b) \times 100 \%$, where As was the OD value of the experimental group (cell-containing medium, CCK-8 reagent, NPs), Ac was the OD value of the control group (cell-containing culture medium, CCK-8 reagent, without NBs), and $\mathrm{Ab}$ was the OD value of the blank group (medium with no cells, no NBs, and no CCK-8 reagent).

\section{Detection of 786-O Cell Apoptosis}

Terminal deoxynucleotidyl transferase (TdT) dUTP nickend labeling (TUNEL) was used to detect apoptosis of 786-O cells. An appropriate amount of exponentially growing 786-O cells was cultured in 24-well plates with sterile cells at $37^{\circ} \mathrm{C}$ and $5 \% \mathrm{CO}_{2}$ for $12 \mathrm{hrs}$ and then divided into 6 groups: a control group, TEM group, TNB group, G250-TNBs group, TNBs+UTND group, and G250-TNBs+UTND group. Except for the control group, the same dose of TEM was used for the other groups, and the final concentration was $2 \mathrm{mM}$. Parameters for ultrasonic irradiation were as follows: frequency of $1 \mathrm{MHz}$, irradiation intensity of $1.0 \mathrm{~W} / \mathrm{cm}^{2}$, and irradiation time of $30 \mathrm{~s} /$ well. After the respective treatments, the cells were continuously cultured at $37^{\circ} \mathrm{C}, 5 \% \mathrm{CO}_{2}$ for $12 \mathrm{hrs}$. The cells were removed, washed 3 times with PBS, and fixed in $4 \%$ paraformaldehyde. The samples were supplemented with $50 \mu \mathrm{L}$ of TUNEL assay solution (Roche Applied Science, Basel, Switzerland), incubated at $37^{\circ} \mathrm{C}$ in the dark for 60 mins, and observed under a fluorescence microscope.

In addition, FITC-labeled recombinant human Annexin $\mathrm{V}$ was used to detect the presence of phosphatidylserine on the cell membrane surface during apoptosis. The 
concentration of 786-O cells in the logarithmic growth phase was adjusted to $5 \times 10^{5} / \mathrm{mL}$. Each well in 6 -well plates was inoculated with $2 \mathrm{~mL}$ cell suspension and incubated for $24 \mathrm{hrs}$. Cells were divided into the aforementioned groups and treated with the corresponding factors before being digested and then washed 3 times with PBS. Cells were resuspended in PBS and centrifuged at $1000 \mathrm{~g}$ for 5 mins. The supernatant was discarded, and the cells were gently resuspended in $195 \mu \mathrm{L}$ Annexin V-FITC binding solution, $5 \mu \mathrm{L}$ Annexin V-FITC (Beyotime Biotechnology, Shanghai, China), and gently mixed, followed by the addition of $10 \mu \mathrm{L}$ propidium iodide staining solution and gentle mixing. They were then incubated at room temperature $\left(20-25^{\circ} \mathrm{C}\right)$ in the dark for $10-20 \mathrm{mins}$ and placed in an ice bath. Subsequently, flow cytometry analysis was performed using a flow cytometer (FACS Vantage SE, BD Biosciences, New York, USA) to detect apoptosis.

\section{In vivo Targeted Binding Ability and Enhancement of Ultrasound Imaging}

To verify the in vivo targeting ability of G250-TNBs and their ability to enhance ultrasound imaging, five nude mice with a tumor volume of $50-100 \mathrm{~mm}^{3}$ were randomly selected for ultrasound imaging on a small animal ultrasound imaging system (Vevo 2100 VisualSonics, Toronto, ON, Canada), scanning with an S250 high-frequency probe. Anesthesia was established using $2 \%$ isoflurane, and the surfaces of the tumors were covered with a coupling agent for energy transduction. The probe was fixed on the maximum cross-section of each tumor xenograft, and the focal center was located at the center of the tumor xenograft tissue. The mice were randomly injected with $200 \mu \mathrm{L}$ of the same concentration of G250-TNBs or TNBs through the orbital venous sinus to collect two-dimensional grayscale images, and then imaging mode was adjusted to contrast-enhanced ultrasound (CEUS) imaging mode (central frequency $20 \mathrm{MHz}$ ), and continuous dynamic images were collected. After the NBs in the mice had completely disappeared, the same method was used to inject $200 \mu \mathrm{L}$ of another type of nanobubbles, and dynamic images were collected. Special software (Vevo 2100 onboard software, VisualSonics, Toronto, ON, Canada) was used to analyze the peak intensity ( $\mathrm{dB}$ value) and duration (time interval between the injection of NBs and completion of CEUS imaging).

\section{Observation of the in vivo Therapeutic Effect}

To evaluate the anti-tumor effect of G250-TNBs combined with UTND in tumor-bearing mice, the mice were randomly assigned to 6 groups $(n=5)$ : control group, TEM group, TNB group, G250-TNBs group, TNBs+UTND group, G250-TNBs+UTND group. The control group received an injection of normal saline ( $0.9 \%$ sodium chloride), and different TEM-containing preparations with the same dose of TEM $(10 \mathrm{mg} / \mathrm{kg})$ were administered to the mice in other groups via tail vein injection once every 2 days. The tumor areas of mice in the TNBs+UTND group and G250-TNBs + UTND group were irradiated using the WED-100 full digital ultrasonic therapy machine. A sufficient amount of coupling agent was used to fill the area between the probe and the tumor xenograft. The probe frequency was $1 \mathrm{MHz}$, and the power was $2.5 \mathrm{~W} / \mathrm{cm}^{2}$. To ensure reperfusion of the tumor area after NB destruction, the built-in $100-\mathrm{Hz}$ pulse of the therapeutic device was combined with the manual " $5 \mathrm{~s}$ on, $5 \mathrm{~s}$ off" mode. ${ }^{13}$ The volume of transplanted tumors in nude mice was recorded on the day treatment started and every 3 days after treatment. After 2 weeks of treatment, all mice were sacrificed, and xenograft tumors were removed. Tumor weight and tumor size were measured to calculate the tumor volume and tumor control rate. ${ }^{16}$ The TUNEL assay was used to evaluate tumor cell apoptosis. The apoptosis index (AI) was calculated as the proportion of positively stained tumor cells among the total number of tumor cells. The total number of tumor cells was counted in 5 randomly selected high-power fields. In addition, hematoxylin and eosin (H\&E)-stained paraffin sections were used to observe histopathological changes in the samples.

\section{Statistical Analysis}

SPSS 23.0 software was used to perform the statistical analysis of the measurement data, which was expressed as $\bar{x} \pm \mathrm{s}$. Data were analyzed using one-way analysis of variance (ANOVA) and paired t-tests. $P<0.05$ represented a significant difference.

\section{Results G250-TNB Characterization}

G250-TNBs were distributed as dispersive dots under a conventional optical microscope (Figure 1A). The concentration of NBs was $(10.09 \pm 1.13) \times 10^{8} / \mathrm{mL}$. NBs presented as uniform spheres with a diameter of approximately $400 \mathrm{~nm}$ under a transmission electron microscope 

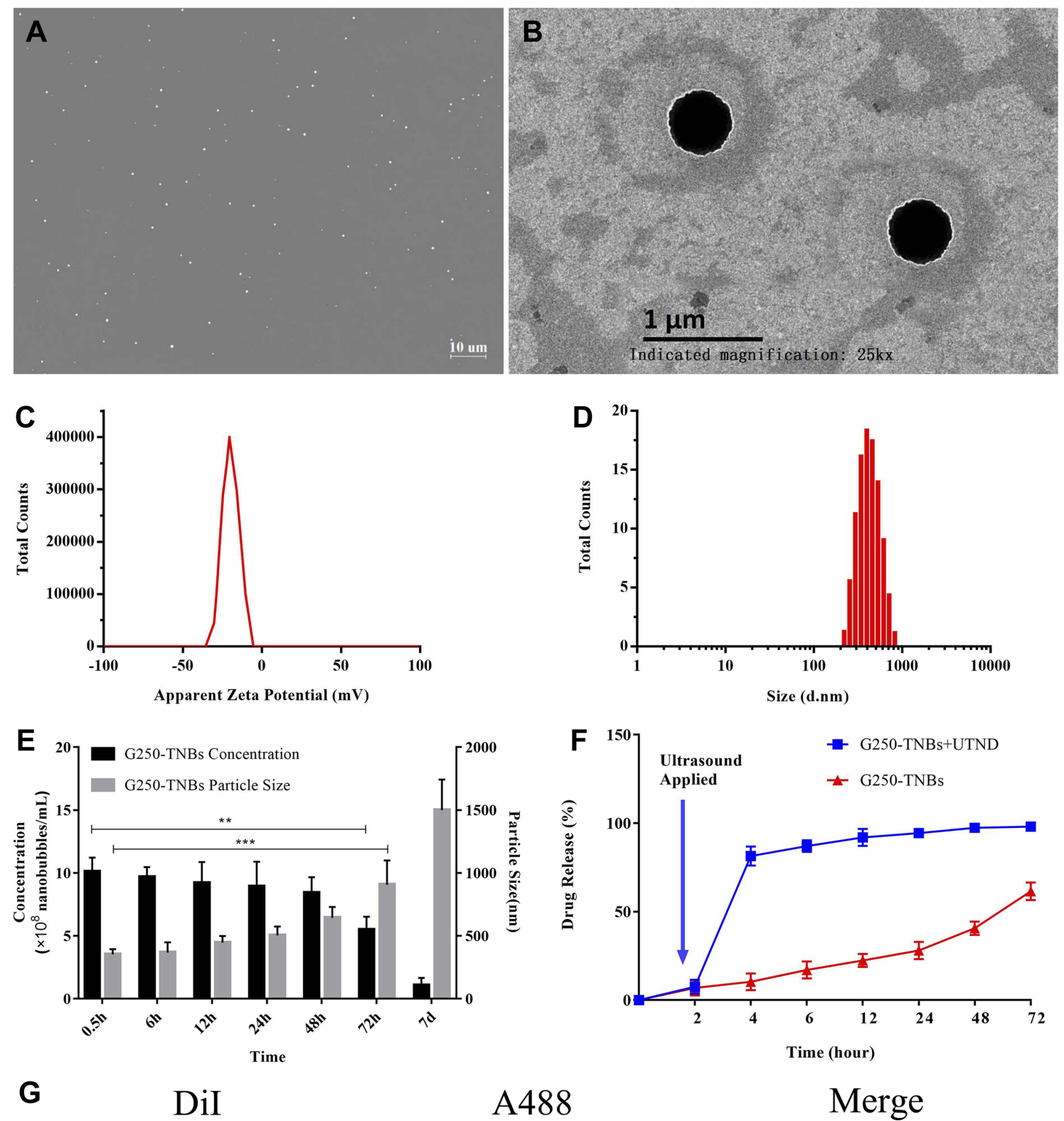

A488
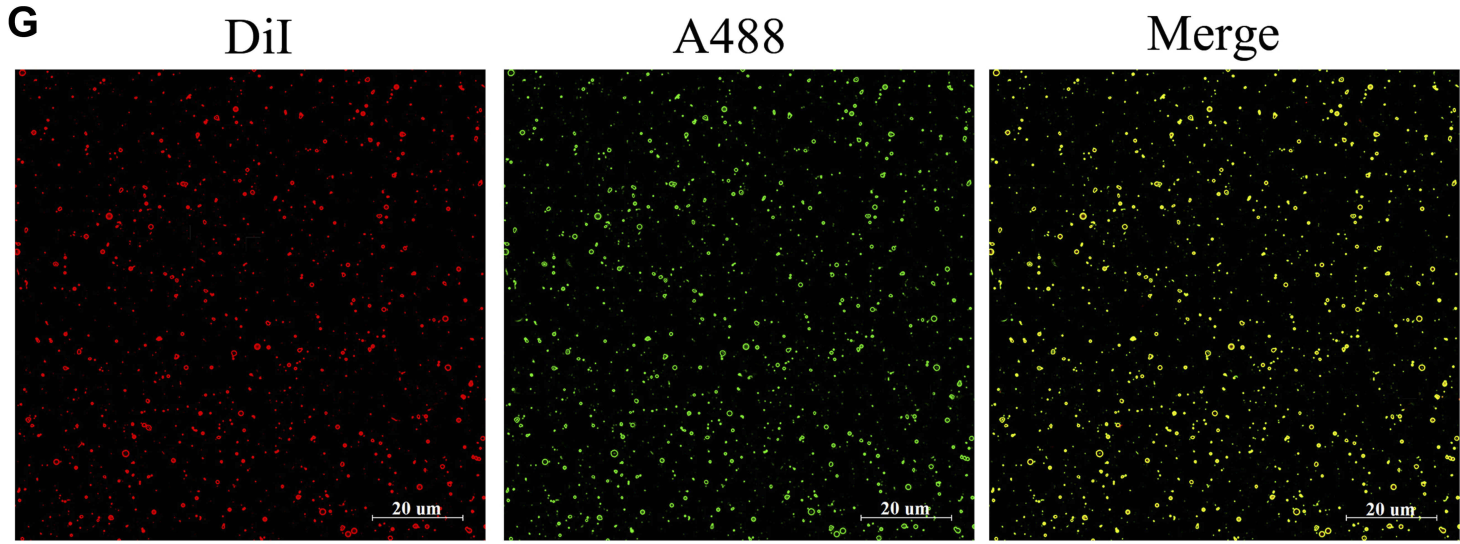

Figure I Characterization of G250-TNBs. (A) Morphology of G250-TNBs under a light microscope. (B) Morphology of G250-TNBs under a transmission electron microscope. (C) Distribution of the zeta potential of G250-TNBs. (D) Distribution of particle diameter of G250-TNBs. (E) Histogram showing the time dependence of changes in the concentration and particle size of the G250-TNBs $(* * \mathrm{P}<0.0 \mathrm{I}$, $* *$ *P $<0.00 \mathrm{I})$. (F) Drug release curves of G250-TNBs. (G) Verification of the coupling of antiG250 nanobodies to drug-loaded NBs by dual fluorescence: Dil-labeled G250-TNBs displayed red fluorescence, Alexa-488-labeled anti-G250 nanobodies showed green fluorescence. Yellow color appeared when the two types of fluorescence overlapped completely, indicating that the G250 nanobodies were ligated to the surfaces of the NBs. 
(Figure 1B). The polydispersity index was $0.0171 \pm$ 0.0063 , and the average zeta potential was $-17.3 \pm 6.9$ $\mathrm{mV}$ (Figure 1C). The mean diameter of G250-TNBs measured using a Malvern Zetasizer Nano ZS90 was $368.7 \pm$ $43.4 \mathrm{~nm}$ (Figure 1D). When stored at $4^{\circ} \mathrm{C}$ until the second day, there was no significant difference in concentration and particle size compared with 0.5 hrs $(\mathrm{P}>0.05)$. However, when they were stored for $72 \mathrm{hrs}$, there was a significant difference in concentration and particle size compared with $0.5 \mathrm{hrs}(\mathrm{P}<0.05)$. On the 7 th day of storage, it could be seen from the appearance that the underlying liquid became cloudy, indicating that some nanobubbles decomposed and broke down. The concentration decreased significantly and the particle size increased significantly (Figure 1E). The EE and LE of G250-TNBs were 68.59\% $\pm 5.43 \%$ and $5.23 \% \pm 0.91 \%$, respectively. The G250TNBs group without ultrasonic irradiation slowly released TEM within $72 \mathrm{hrs}$. When ultrasonic irradiation was applied to G250-TNBs, the TEM carried by the G250TNBs was almost completely released in a short time, indicating that UTND could achieve the purpose of rapid drug release. The ultrasound-mediated cavitation effect caused the rupture of TNBs and promoted drug release
(Figure 1F). Coupling between drug-loaded NBs and antiG250 nanobodies was a prerequisite to achieve the targeting ability. Under CLSM, the Dil-labeled G250-TNBs showed red fluorescence, while the Alexa-488-labeled anti-G250 nanobodies exhibited green fluorescence. The red and green fluorescence completely overlapped (Figure 1G), indicating that anti-G250 nanobodies were coupled uniformly on the surfaces of TNBs. This result confirmed that G250-NBs were successfully constructed.

\section{In vitro Targeted Binding Ability of G250- TNBs}

Under CLSM, it was found that Dil-labeled G250-TNBs bound to 786-O cells that expressed G250 antigen in large quantities, while Dil-labeled TNBs almost did not bind to 786-O cells. After being treated with anti-G250 nanobodies, the G250 antigen on the surfaces of 786-O cells bound to the anti-G250 nanobodies, and the 786-O cells no longer bound to Dil-labeled G250-TNBs (Figure 2). These results indicated that G250-TNBs could specifically bind to 786-O cells expressing G250 antigens.
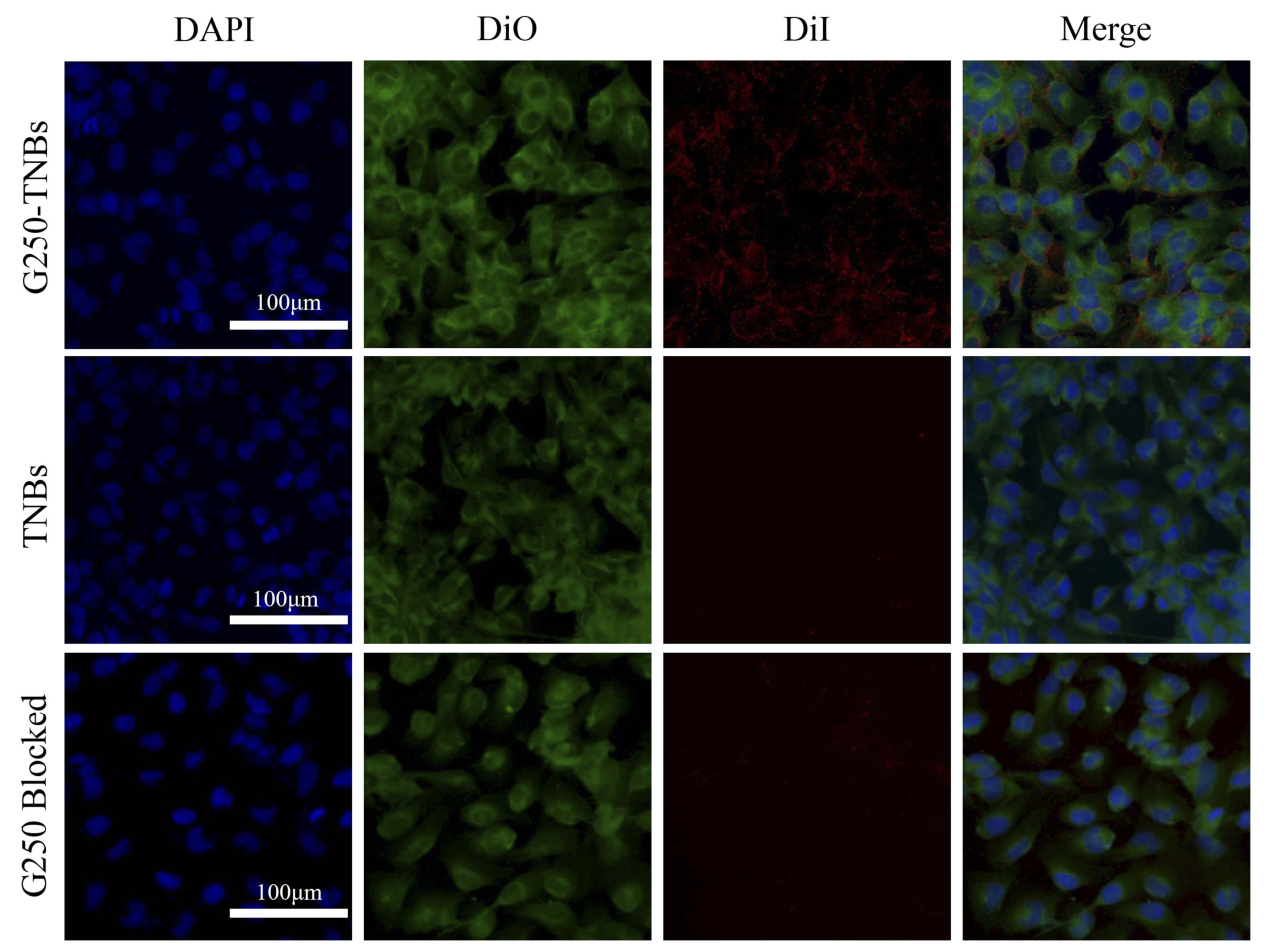

Figure 2 In vitro targeted binding ability of G250-TNBs to 786-O cells. DAPI-stained nuclei (blue), DiO-stained cell membrane (green), Dil-labeled NBs (red). It can be seen that a large amount of Dil-labeled G250-TNBs bound to 786-O cells (the first row), Dil-labeled TNBs did not bind to 786-O cells (the second row), and Dil-labeled G250TNBs did not bind to anti-G250 nanobody-treated $786-0$ cells (the third row). 


\section{In vitro Cytotoxicity of Combined} Treatment of G250-TNBs and Ultrasonic Irradiation

The CCK-8 assay was used to test the in vitro cytotoxicity of the combined treatment of G250-TNBs and ultrasonic irradiation (Figure 3 ). The results showed that the cell survival rate of the G250-TNBs+UTND group was significantly lower than that of the other groups $(\mathrm{P}<0.05)$, indicating that the G250-TNBs combined with UTND could achieve the highest antiproliferative efficiency. The cell survival rate of the TNBs+UTND group is significantly higher than that of G250-TNBs+UTND group ( $\mathrm{P}<0.05)$, demonstrating that the specific targeting ability of anti-G250 nanobodies to 786-O cells increased the accumulation of G250-TNBs around cells, and the increase in the local drug concentration under the action of UTND further enhanced the killing effect on tumor cells. While without UTND, the cell survival rate of the TNB group and G250-TNBs group was significantly higher than that of the TNBs+ UTND group and G250-TNBs+UTND group, indicating that UTND could promote drug release and significantly enhance cell proliferation inhibition. The cell survival rate of the TEM group was lower than that of the G250-TNB group, but higher than that of the G250-TNBs + UTND group, showing that without ultrasonic irradiation, drug release of G250-TNBs was much slower, local drug concentration was not as high as that of direct use of TEM, and local drug concentration after ultrasonic irradiation was

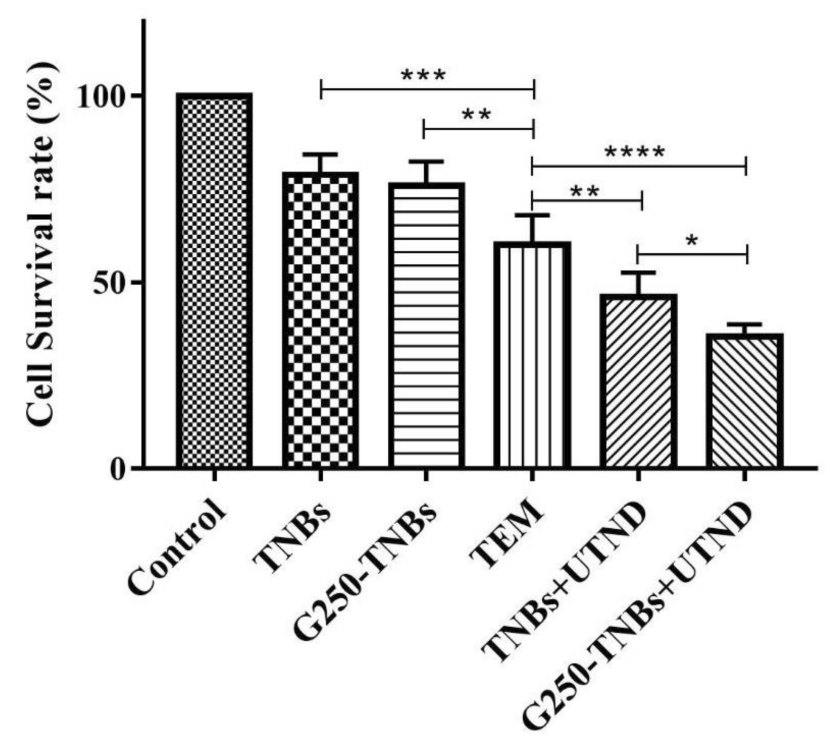

Figure 3 Detection of the cell survival rate of tumor cells under combined treatment with G250-TNBs and ultrasonic irradiation against 786-O cells using CCK-8. $* P<0.05$, $* * P<0.01$, $* * * P<0.001$, and $* * * * P<0.0001$. significantly higher than that of direct use of TEM. These results indicated that both anti-G250 nanobodies and UTND played important roles in the antiproliferation of RCC cells.

To study the synergistic effect of G250-TNBs and UTND in inducing apoptosis of 786-O cells, TUNEL staining was performed (Figure 4A). The number of cells stained in each field (apoptotic cells) was randomly selected for analysis (Figure 4B). The results showed that the apoptosis rate of 786-O cells in the TEM group was higher than in the TNBs and G250-TNBs groups $(\mathrm{P}<0.001)$ but lower than in the TNBs+UTND and G250-TNBs+UTND groups ( $\mathrm{P}<0.0001)$; most importantly, the apoptosis rate was significantly higher in the G250-TNBs+UTND group than the TNBs+UTND group ( $\mathrm{P}<0.0001)$. In addition, flow cytometry was used to measure the apoptosis rate in each group to further study the synergistic therapeutic efficiency of G250-TNBs and ultrasonic irradiation (Figure 4C). The apoptosis rate was highest in the G250-TNBs+UTND group (Figure 4D), showing that the combined G250-TNBs and ultrasonic irradiation treatment was the most effective, and the results were statistically significant. Without ultrasonic irradiation, the therapeutic effect was higher in the TEM group than in the TNBs and G250-TNBs groups. The TNBs+UTND and G250-TNBs +UTND groups, which were treated with ultrasonic irradiation, showed a significantly better therapeutic effect than the TEM group. This result was consistent with the TUNEL staining results.

\section{In vivo Targeted Binding Ability and Enhancement of Ultrasound Imaging}

Prior to injection of NBs, B-mode ultrasound and contrastenhanced ultrasound (CEUS) were used to scan the transplanted tumors. The ultrasonograms of the tumors showed regular hypoechoic patterns (Figure $5 \mathrm{~A}$ and $\mathrm{B}$ ). After the injection of drug-loaded NBs, the contrast of the ultrasonogram at the tumor site was significantly enhanced (Figure 5C), indicating that the drug-loaded NBs could pass through the tumor blood vessels and enter the tumor tissue space. The area under the curve (AUC) was calculated by plotting the time-intensity curve between the peak time and 10 mins (Figure 5D). The AUC after injection of G250-TNBs was significantly different from the AUC after the injection of TNBs $(P<0.01)$ (Figure 5E). These experimental results suggested that, compared with TNBs, G250-TNBs could actively target tumor tissues, and NBs could accumulate at tumor sites to obtain hyperechoic signals and contrast-enhanced ultrasonography. 


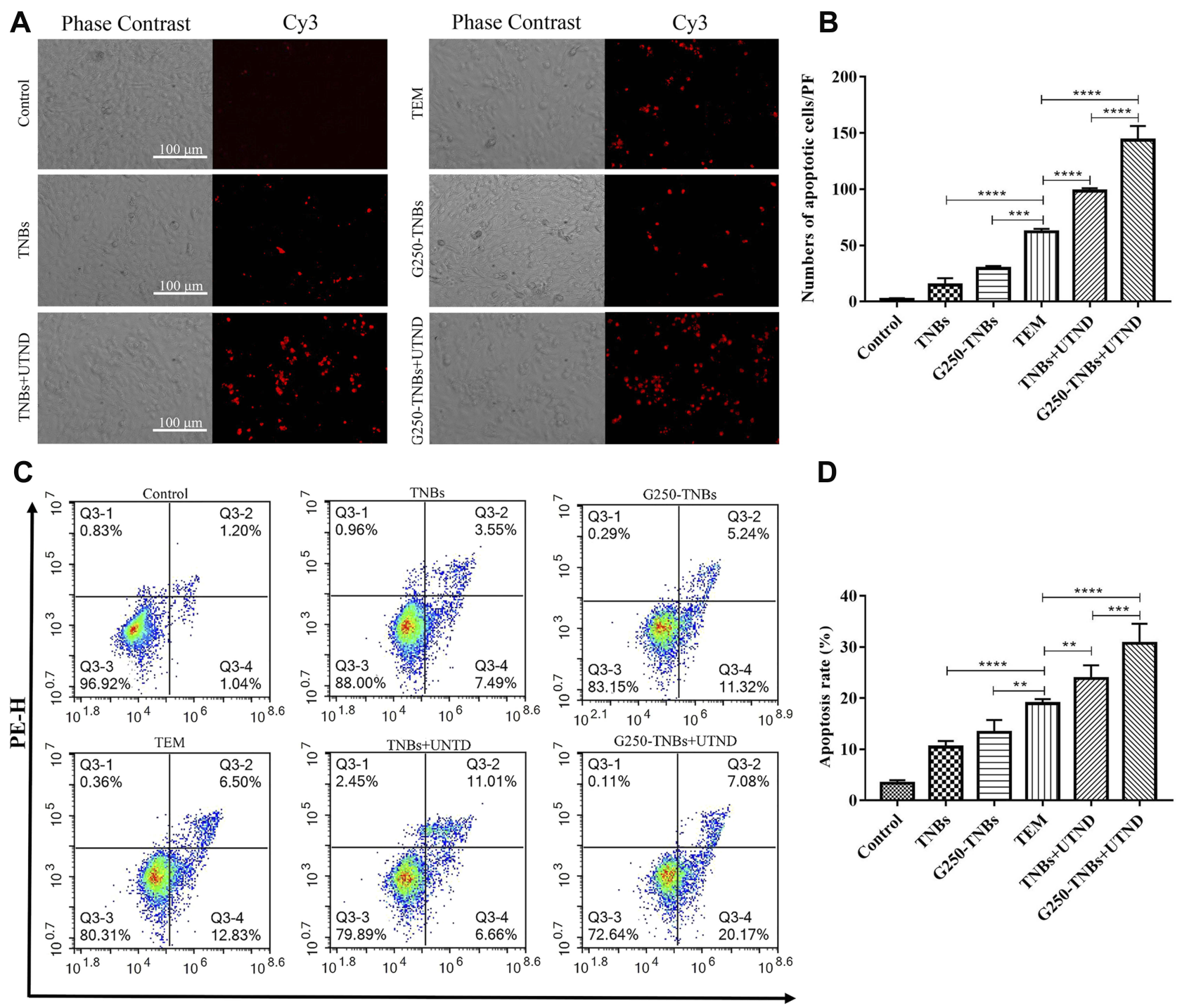

FITC-H

Figure 4 Detection of tumor cell apoptosis. (A and B) Apoptosis of tumor cells in each group detected by the one-step TUNEL assay. (A) Red indicates Cy3-labeled apoptotic cells. (B) The counts of apoptotic cells in each group under each microscopic field $(* * * P<0.001$, $* * * * P<0.0001)$. (C and $\mathbf{D})$ The apoptosis rate in each group detected using a flow cytometer. (D) The apoptosis rate in each group $(* * P<0.01, * * * P<0.001, * * * * P<0.0001)$.

\section{In vivo Therapeutic Effect}

To evaluate the combined therapeutic effect of G250TNBs and UTND in xenograft tumors in nude mice, the volume and quality of xenograft tumors were measured after grouping and treatment. The results showed that the mean volume of xenograft tumors in the G250TNBs+UTND group was smallest $(\mathrm{P}<0.05)$, and compared with the control group, the tumor growth inhibition rate reached $97.56 \%$ (Table 1). As shown in Figure 6, the volume and mass of xenograft tumors were higher in the TNB group and G250-TNBs group than in the TEM group $(\mathrm{P}<0.001)$, while the volume and mass of xenograft tumors were significantly smaller in the TNBs+UTND and G250-TNBs+UTND groups than the TEM group $(\mathrm{P}<0.0001)$. This result suggested that the anti-tumor efficiencies were significantly higher in the TNBs+UTND and G250-TNBs+UTND groups than the TNBs and G250-TNBs groups, respectively. More importantly, the volume and mass of xenograft tumors were significantly smaller in the G250-TNBs + UTND group than the TNBs+UTND group $(\mathrm{P}<0.05)$. This phenomenon indicated that anti-G250 nanobodies were conducive to the aggregation of TNBs at the tumor site and the release of TEM from TNBs under the action 


\section{CEUS}
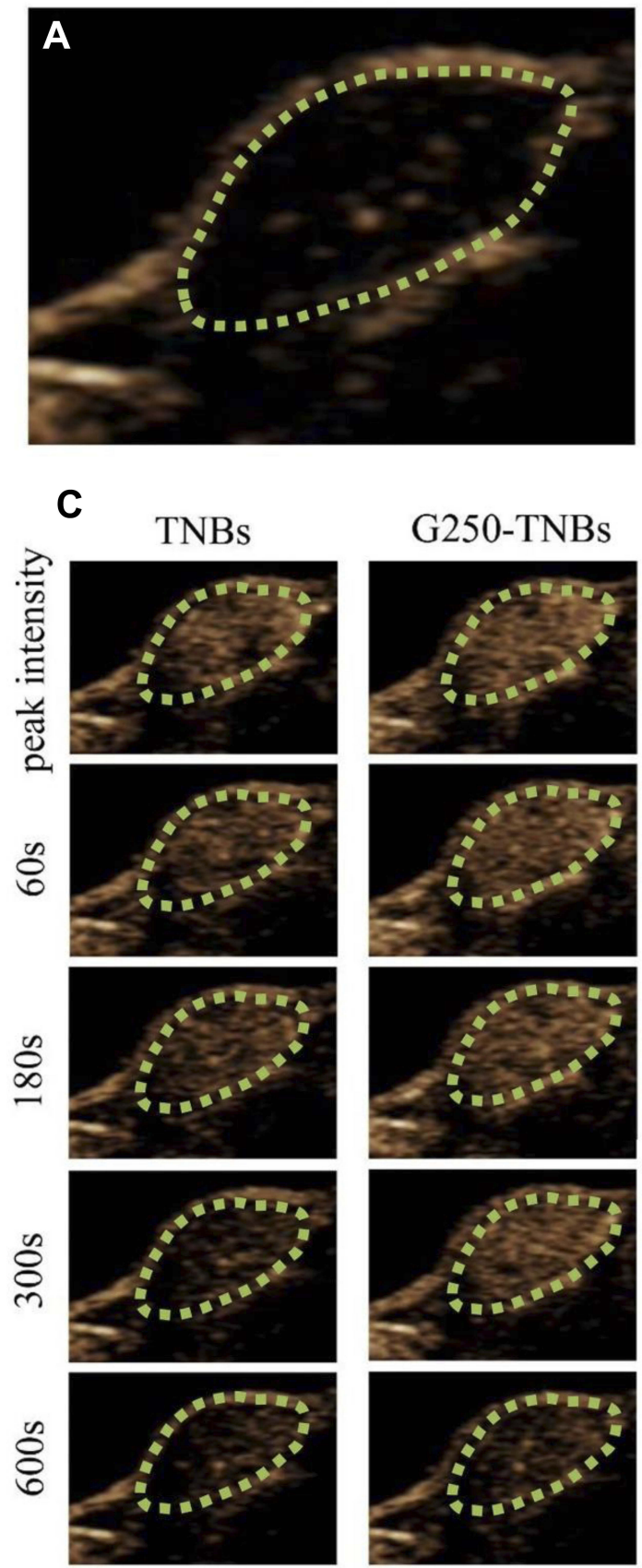

\section{G250-TNBs}
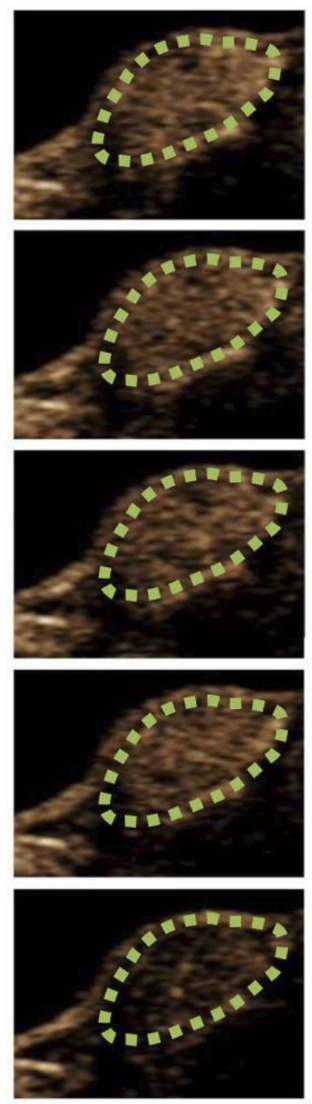

\section{B-Mode}

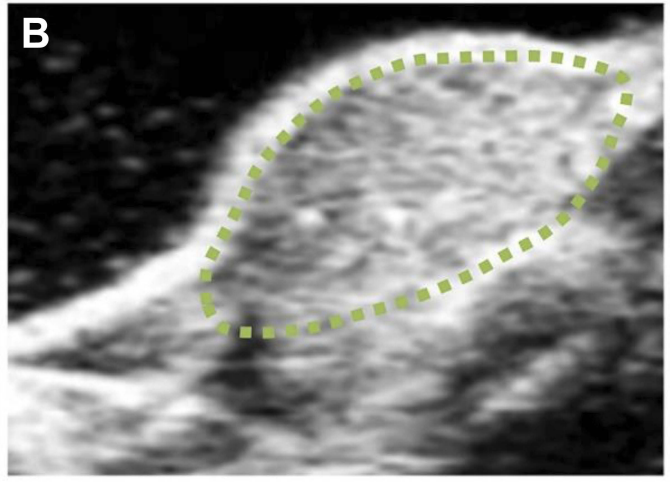

D

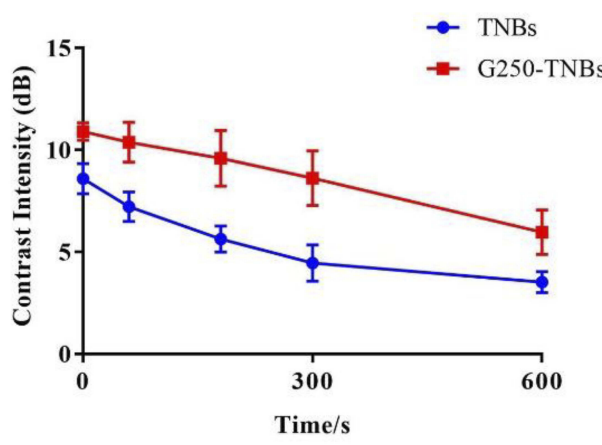

E

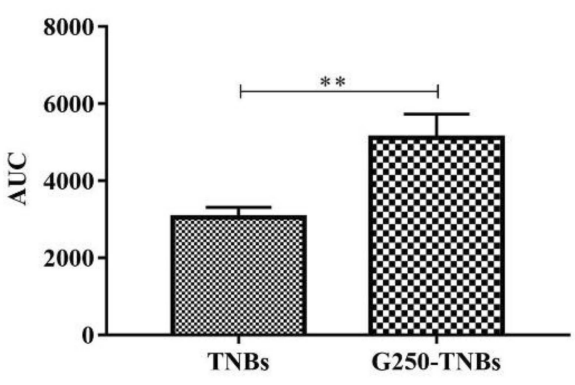

Figure $\mathbf{5}$ The in vivo targeted binding ability of drug-loaded NBs and ultrasonography. The green dotted circles represented areas of xenograft tumors. (A and B) the contrast-enhanced ultrasound (CEUS) and B-mode images of the tumor site. (C) the continuous recording of the CEUS image of the tumor tissue after injection of the drugloaded NBs. (D) the time-ultrasonic intensity curve of the tumor tissue after injection of the two types of drug-loaded NBs. (E) The areas under the curve were calculated from the time-ultrasonic intensity curves and statistically analyzed (**P 0.01 ).

of UTND, further enhancing the anti-tumor efficacy. These results were consistent with the in vitro study results.

H\&E staining was performed to evaluate the histological characteristics of RCC xenografts after treatment with various methods (Figure $7 \mathrm{~A}-\mathrm{F}$ ). H\&E staining of tumor tissues in the control group revealed a normal cell morphology, while a large number of lysed cell membrane and nucleus fragments were observed in the G250-TNBs +UTND group. TUNEL staining was used to evaluate apoptosis in tissue sections, where the stained apoptotic cell nucleus was brown (Figure $7 \mathrm{G}-\mathrm{L}$ ) and to calculate the 
Table I Mean Tumor Volume and Mean Percentage Tumor Inhibition in Each Group After Treatment for 20 Days (mean \pm SD, $n=5$ )

\begin{tabular}{|l|l|l|}
\hline Group & $\begin{array}{l}\text { Tumor Volume } \\
\left(\mathbf{m m}^{\mathbf{3}}\right)\end{array}$ & $\begin{array}{l}\text { Mean Tumor Inhibition } \\
\text { Rate }(\%)\end{array}$ \\
\hline Control & $854.74 \pm 108.32$ & - \\
TNBs & $563.48 \pm 44.65^{*, \Delta}$ & 34.076 \\
G250-TNBs & $516.07 \pm 70.99^{*, \Delta}$ & 39.62 \\
TEM & $342.60 \pm 28.67^{*, \Delta}$ & 59.92 \\
TNBs+UTND & $140.09 \pm 20.55^{*, \Delta}$ & 83.61 \\
G250-TNBs & $20.84 \pm 6.34^{*}$ & 97.56 \\
+UTND & & \\
\hline
\end{tabular}

Notes: $* P<0.05$ compared with the control group; ${ }^{\Delta} P<0.05$ compared with the G250-TNBs+UTND group.

apoptosis index (Figure 7M). The most significant apoptosis of tumor cells occurred in the G250-TNBs+UTND group $(P<0.05)$. These results were consistent with the H\&E staining results. Therefore, this part of the experimental results suggested that the therapeutic effect was significantly greater in the G250-TNBs+UTND group than the other treatment groups.

\section{Discussion}

The incidence of RCC is increasing each year. Because the symptoms are not obvious at the early stage, when typical symptoms of renal cancer occur, such as hematuria, back pain, and weight loss in a short period of time, it is already at an advanced stage. The sensitivity of late-stage RCC to chemotherapy is low, and the effective rate is approximately $6 \%$. Chemotherapy often cannot prolong the survival of patients with late-stage RCC. In response to the unique pathogenesis of RCC, scientists and clinicians have started to introduce molecular targeted drugs in RCC therapy. These drugs include VEGFR inhibitors, mTOR inhibitors, and bevacizumab, which have greatly prolonged the survival of RCC patients, greatly improved quality of life, and become the first-line drug in the treatment of RCC. ${ }^{17}$ However, clinical applications have shown that molecular targeted drugs still have greater side effects involving the skin, digestive tract, heart, lung, and the like, which are caused by targeted drugs acting outside the target organ. These are correlated with the systemic administration of the targeted drugs. Many patients discontinue treatment

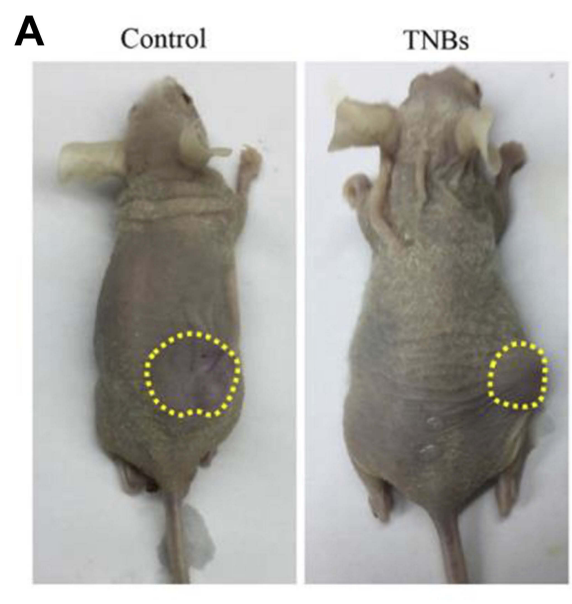

B

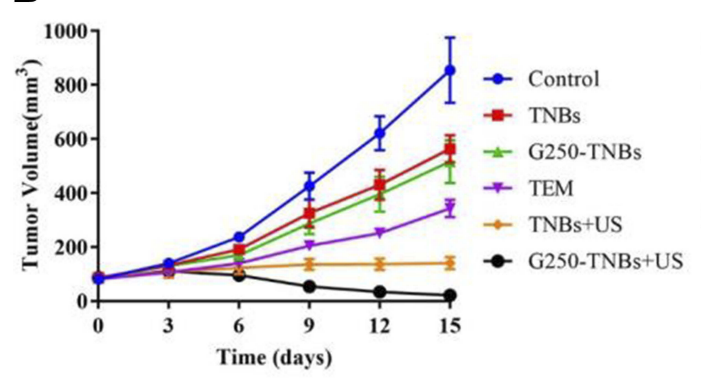

G250-TNBs

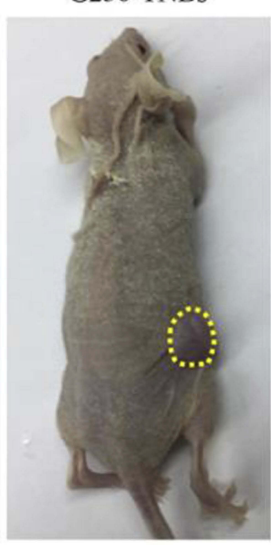

C
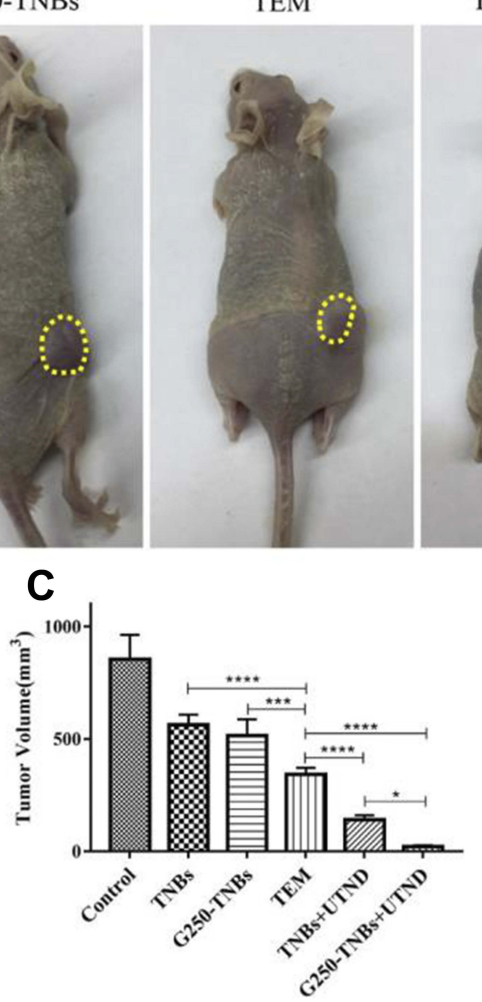

TNBs+UTND

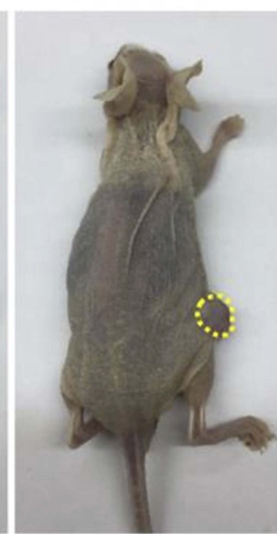

D

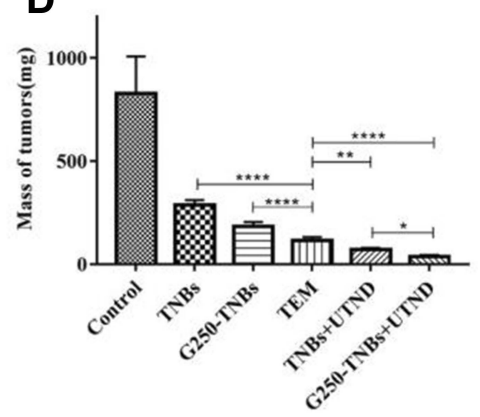

G250-TNBs+UTND

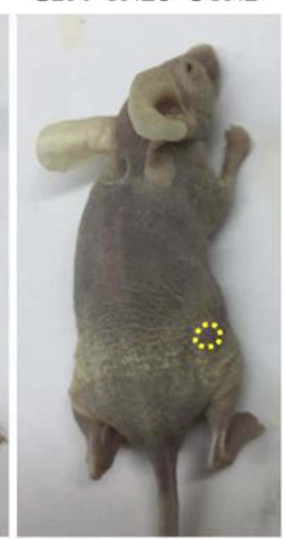

$\because$

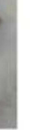

\section{.}



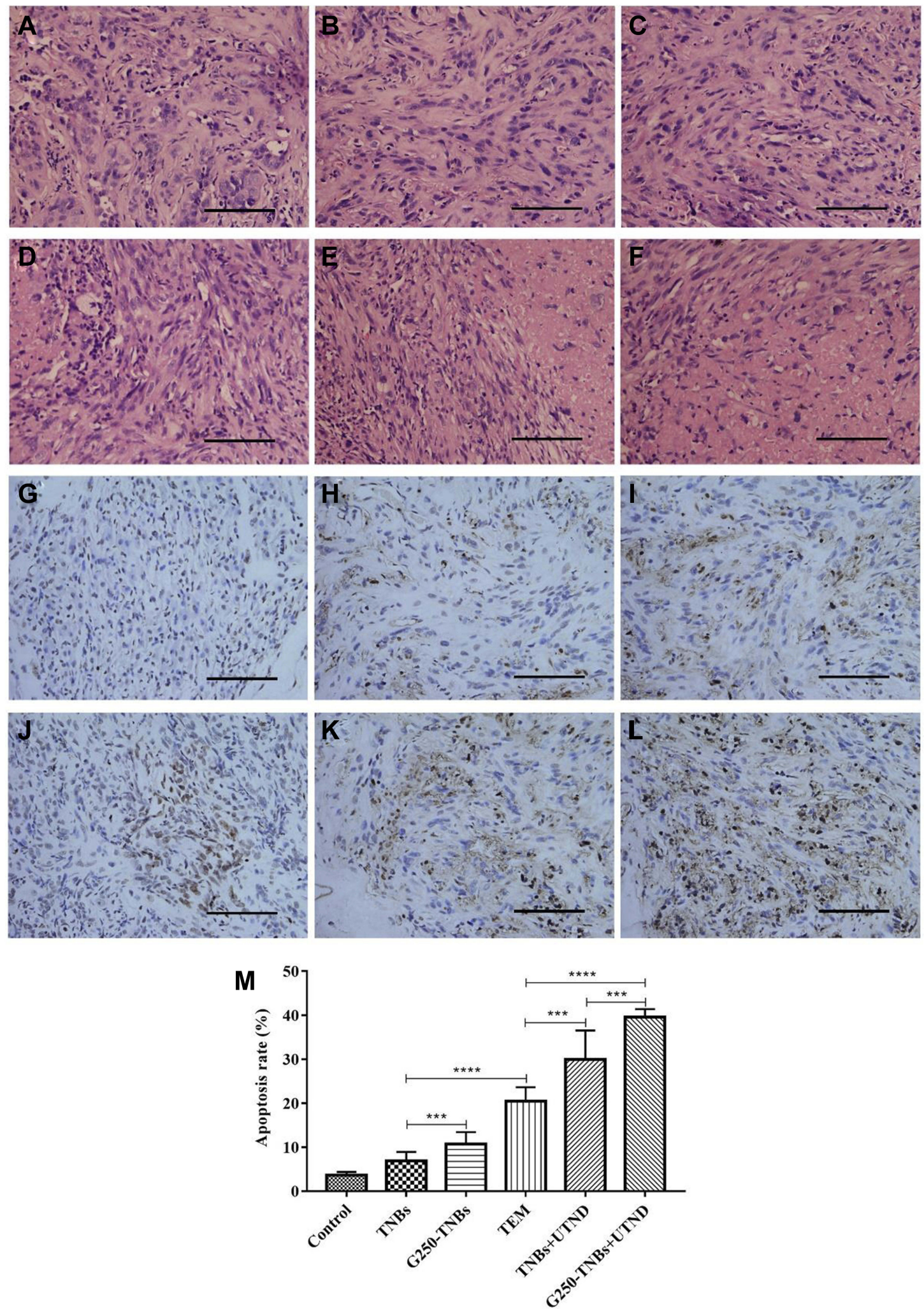

Figure 7 Immunohistochemical analysis of the xenograft tumor tissue. (A-F) H\&E staining results of the control group, TNB group, G250-TNBs group, TEM group, TNBs +UTND group, and G250-TNBs+UTND group, respectively. (G-L) TUNEL staining results of the control group, TNB group, G250-TNBs group, TEM group, TNBs+UTND group, and G250-TNBs+UTND group, respectively. Scale: $100 \mu \mathrm{m} ;(\mathbf{M})$ the apoptosis index for each group of tumor tissues $(* * * P<0.00 \mathrm{I}, * * * * P<0.000 \mathrm{I})$. 
because they cannot tolerate the side effects. Therefore, it is imperative to change the mode of drug administration to reduce the side effects. TDDS has provided the possibility of solving this problem. The ultrasound-nanobubble TDDS has become a cutting-edge technology in modern cancer therapies because it can change the pharmacokinetics of drugs, increase the efficiency of drug action, and reduce the side effects of drugs ${ }^{18}$. As an indirect targeted drug delivery method, targeted NBs allow anti-tumor drugs to accumulate locally in tumors and increase the payload of cytotoxic drugs. While improving the treatment efficacy of drugs, NBs as drug carriers can prevent drug degradation and reduce their accumulation in normal cells, thereby reducing toxic and side effects of drugs in the body. 19

Currently, there are few reports on TDDS for RCC. Our experiments successfully constructed the anti-G250 nanobody-mediated nanobubble TDDS. The G250 antigen is expressed in most RCC cells but not in normal kidney tissues. ${ }^{20}$ Our study confirmed that the G250TNBs could specifically bind to 786-O cells expressing G250 antigens and the specific targeting ability of antiG250 nanobodies to 786-O cells could increase the accumulation of G250-TNBs around cells. Therefore, the constructed drug carrier G250-TNB can increase the active targeting of drugs. Moreover, since the nonintegrity of new tumor blood vessel walls allows the traversal of NBs with a diameter less than $700 \mathrm{~nm},{ }^{9}$ the G250-TNBs constructed in this study had a relatively uniform diameter of approximately $368.7 \mathrm{~nm}$, allowing them to pass freely through tumor blood vessels to enter the tumor tissue, thereby reducing damage to normal tissues and cells.

There have been many studies on nanobubbles as drug carriers, but low drug loading efficiency is still a common problem. The drug loading efficiency of some hydrophobic drugs, such as paclitaxel (PTX) and doxorubicin (DOX), was ranged from $2.666 \pm 0.092 \%$ to $6.69 \pm 0.69 \%$ in the literature, ${ }^{21-25}$ and the drug loading efficiency of G250TNBs in this study was $5.23 \% \pm 0.91 \%$. On the one hand, the low efficiency of drug loading may be due to the fact that the nanobubbles are vesicles with the perfluoropropane gas wrapped in the lipid envelope, and TEM is a hydrophobic drug that encapsulates only in the lipid envelope. On the other hand, the small size of NBs limits the drug-carrying capacity. Obviously, the larger the diameter, the higher the drug loading efficiency and the better the anti-tumor treatment effect. However, large diameter bubbles could not pass through the endothelial space of tumor vessels to enter the tumor tissue space, which was not conducive to local drug accumulation. ${ }^{26}$

By comparing our experimental results, we found that the TEM group exhibited significantly superior tumor control and apoptosis promotion than the G250TNBs group, potentially because drugs carried by G250TNBs were released more slowly within a certain period of time due to the better stability of G250-TNBs. Therefore, the G250-TNBs group had a lower local TEM concentration than the TEM group, and direct use of TEM facilitated the passive diffusion, internalization, penetration, and entry into cells. ${ }^{27}$ So the treatment efficacy of G250-TNBs was inferior to that of TEM alone. However, direct use of TEM has a limited clinical application due to its associated adverse reactions, so how to enhance the drug release of G250TNBs becomes particularly important.

Therefore, we combined ultrasound to improve the therapeutic effect of G250-TNBs. Under the action of UTND, drug-loaded NBs ruptured, and the drug was released locally in the tumor, thereby increasing the local concentration of the drug. Moreover, the cavitation effect of ultrasound increased the permeability of the cell membrane and facilitated the entry of the drug into cells, greatly improving the therapeutic effect of the drugs. ${ }^{28-31}$ The experimental results confirmed that G250-TNBs combined with UTND more significantly inhibited the proliferation of tumor cells and promoted their apoptosis than G250-TNBs alone, and showed a superior effect compared with TEM alone $(P<0.05)$. This phenomenon is consistent with previous studies, ${ }^{15}$ that is, UTND can cause the concentrated release of drugs in NBs, thereby improving the anti-proliferative effect of drugs while reducing the influence of drugs on the surrounding normal cells and reducing their side effects. The results of in vivo studies in mice with RCC xenografts further elucidated that the growth of the transplanted tumors was significantly reduced after combined treatment with G250-TNBs and UTND, and the experimental results of $\mathrm{H} \& \mathrm{E}$ and immunohistochemistry further confirmed that the apoptosis rate of tumor cells was higher in the G250TNBs+UTND group $(P<0.05)$. These results further indicated that under the action of UTND, such as ultrasoundmediated cavitation effect, nanobubble rupture, drug release and mechanical action, the local concentration of TEM was significantly increased in tumor tissues, tumor 
cell apoptosis was promoted, and the action efficiency and intensity of the TDDS were improved.

\section{Conclusion}

In summary, our experimental results suggest that G250TNBs combined with UTND can deliver anti-tumor drugs to local RCC cells and increase local effective drug concentrations in tumors, thus enhancing anti-tumor efficacy and reducing side effects of drugs. This approach provides a potential novel method for targeted RCC therapy in clinical practice. With further research, targeted drug-loaded NBs combined with UTND will gradually be used in clinical applications for the treatment of RCC.

\section{Acknowledgments}

This work was supported by the National Natural Science Foundation of China (No. 81472399).

\section{Disclosure}

The authors declare that they have no competing interests in this work.

\section{References}

1. Capitanio U, Montorsi F. Renal cancer. Lancet. 2016;387:894-906. doi:10.1016/S0140-6736(15)00046-X

2. Minguet J, Smith KH, Bramlage CP, et al. Targeted therapies for treatment of renal cell carcinoma: recent advances and future perspectives. Cancer Chemother Pharmacol. 2015;76:219-233. doi:10.1007/ s00280-015-2770-3

3. Ljungberg B, Bensalah $\mathrm{K}$, Canfield $\mathrm{S}$, et al. EAU guidelines on renal cell carcinoma: 2014 update. Eur Urol. 2015;67:913-924. doi:10.1016/j.eururo.2015.01.005

4. Bergmann L, Maute L, Guschmann M. Temsirolimus for advanced renal cell carcinoma. Expert Rev Anticancer Ther. 2014;14:9-21. doi:10.1586/14737140.2014.864562

5. Zatovicova M, Jelenska L, Hulikova A, et al. Monoclonal antibody G250 targeting CA: binding specificity, internalization and therapeutic effects in a non-renal cancer model. Int J Oncol. 2014;45:2455-2467. doi:10.3892/ijo.2014.2658

6. Ming H, Luofu W, Jun J, et al. Preparation and identification of camel nanobodies against human G250 ectodomain. $J$ Third Mil Med Uni. 2017;39:541-547.

7. Kijanka M, Dorresteijn B, Oliveira S, et al. Nanobody-based cancer therapy of solid tumors. Nanomedicine. 2015;10:161-174. doi: $10.2217 / \mathrm{nnm} .14 .178$

8. Iqbal J, Anwar F, Afridi S. Targeted drug delivery systems and their therapeutic applications in cancer and immune pathological conditions. Infect Disord Drug Targets. 2017;17:149-159. doi:10.2174/ 1871526517666170606102623

9. Wang L, Zhang M, Tan K, et al. Preparation of nanobubbles carrying androgen receptor siRNA and their inhibitory effects on androgen-independent prostate cancer when combined with ultrasonic irradiation. PLoS One. 2014;9(5):e96586. doi:10.1371/journal. pone. 0096586
10. Zhu L, Guo Y, Wang L, et al. Construction of ultrasonic nanobubbles carrying CAIX polypeptides to target carcinoma cells derived from various organs. J Nanobiotechnol. 2017;15:63. doi:10.1186/s12951017-0307-0

11. Yang H, Cai W, Xu L, et al. Nanobubble-affibody: novel ultrasound contrast agents for targeted molecular ultrasound imaging of tumor. Biomaterials. 2015;37:279-288. doi:10.1016/j.biomaterials.2014.10.013

12. Theek B, Baues M, Ojha T, et al. Sonoporation enhances liposome accumulation and penetration in tumors with low EPR. $J$ Control Release. 2016;231:77-85. doi:10.1016/j.jconrel.2016.02.021

13. Fan X, Wang L, Guo Y, et al. Inhibition of prostate cancer growth using doxorubicin assisted by ultrasound-targeted nanobubble destruction. Int J Nanomed. 2016;11:3585-3596. doi:10.2147/IJN. S111808

14. Tian Y, Liu Z, Zhang L, et al. Apatinib-loaded lipid nanobubbles combined with ultrasound-targeted nanobubble destruction for synergistic treatment of HepG2 cells in vitro. Onco Targets Ther. 2018;11:4785-4795. doi:10.2147/OTT

15. Cao Y, Chen Y, Yu T, et al. Drug release from phase-changeable nanodroplets triggered by low-intensity focused ultrasound. Theranostics. 2018;8:1327-1339. doi:10.7150/thno.21492

16. Gong Y, Wang Z, Dong G, et al. Low-intensity focused ultrasound mediated localized drug delivery for liver tumors in rabbits. Drug Deliv. 2016;23:2280-2289. doi:10.3109/10717544.2014.972528

17. Calvo E, Porta C, Grunwald V, et al. The current and evolving landscape of first-line treatments for advanced renal cell carcinoma. Oncologist. 2019;24:338-348. doi:10.1634/theoncologist.2018-0267

18. Huang P, Wang X, Liang X, et al. Nano-, micro-, and macroscale drug delivery systems for cancer immunotherapy. Acta Biomater. 2019;85:1-26. doi:10.1016/j.actbio.2018.12.028

19. Pérez-Herrero E, Fernández-Medarde A. Advanced targeted therapies in cancer: drug nanocarriers, the future of chemotherapy. Eur $J$ Pharm Biopharm. 2015;93:52-79. doi:10.1016/j.ejpb.2015.03.018

20. Mahon BP, M A P, McKenna R. Targeting carbonic anhydrase IX activity and expression. Molecules. 2015;20:2323-2348. doi:10.3390/ molecules 20022323

21. Ma J, Shen M, Xu CS, et al. Biodegradable double-targeted PTXmPEG-PLGA nanoparticles for ultrasound contrast enhanced imaging and antitumor therapy in vitro. Oncotarget. 2016;7(48):8000880018. doi:10.18632/oncotarget.13243

22. Wu M, Wang Y, Wang Y, et al. Paclitaxel-loaded and A10-3.2 aptamer-targeted poly(lactide-co-glycolic acid) nanobubbles for ultrasound imaging and therapy of prostate cancer. Int J Nanomed. 2017;12:5313-5330. doi:10.2147/IJN.S136032

23. Liu F, Chen Y, Li Y, et al. Folate-receptor-targeted laser-activable poly(lactide-co-glycolic acid) nanoparticles loaded with paclitaxel/ indocyanine green for photoacoustic/ultrasound imaging and chemo/ photothermal therapy. Int $J$ Nanomed. 2018;13:5139-5158. doi: $10.2147 /$ IJN.S167043

24. Prabhakar A, Banerjee R. Nanobubble liposome complexes for diagnostic imaging and ultrasound-triggered drug delivery in cancers: a theranostic approach. ACS Omega. 2019;4:15567-15580. doi:10.1021/ acsomega.9b01924

25. Kuo TT, Wang CH, Wang JY, et al. Concurrent osteosarcoma theranostic strategy using contrast-enhanced ultrasound and drug-loaded bubbles. Pharmacentics. 2019;11(5). doi:10.3390/pharmaceutics 11050223

26. Jain A, Tiwari A, Verma A, et al. Ultrasound-based triggered drug delivery to tumors. Drug Deliv Transl Res. 2018;8:150-164. doi:10.1007/s13346-017-0448-6

27. Xing L, Shi Q, Zheng K, et al. Ultrasound-mediated microbubble destruction (UMMD) facilitates the delivery of CA19-9 targeted and paclitaxel loaded mPEG-PLGA-PLL nanoparticles in pancreatic cancer. Theranostics. 2016;6:1573-1587. doi:10.7150/thno.15164

28. Chen H, Hwang J. Ultrasound-targeted microbubble destruction for chemotherapeutic drug delivery to solid tumors. $J$ Ther Ultrasound. 2013;1:10. doi:10.1186/2050-5736-1-10 
29. Tayier B, Deng Z, Wang Y, et al. Biosynthetic nanobubbles for targeted gene delivery by focused ultrasound. Nanoscale. 2019;11:14757-14768. doi:10.1039/C9NR03402A

30. Andrews LE, Chan MH, Liu RS. Nano-lipospheres as acoustically active ultrasound contrast agents: evolving tumor imaging and therapy technique. Nanotechnology. 2019;30:182001. doi:10.1088/13616528/aafeb9
31. de Leon A, Perera R, Nittayacharn P, et al. Ultrasound contrast agents and delivery systems in cancer detection and therapy. Adv Cancer Res. 2018;139:57-84.

\section{Publish your work in this journal}

The International Journal of Nanomedicine is an international, peerreviewed journal focusing on the application of nanotechnology in diagnostics, therapeutics, and drug delivery systems throughout the biomedical field. This journal is indexed on PubMed Central, MedLine, CAS, SciSearch ${ }^{\circledR}$, Current Contents ${ }^{\circledR} /$ Clinical Medicine,
Journal Citation Reports/Science Edition, EMBase, Scopus and the Elsevier Bibliographic databases. The manuscript management system is completely online and includes a very quick and fair peer-review system, which is all easy to use. Visit http://www.dovepress.com/ testimonials.php to read real quotes from published authors. 